\title{
Tobacco Industry Branding Strategies and Its Influence on Young Adults
}

\author{
Umme Salma Anee*, H M Miraz Mahmud, Anika Islam, Mohammad Shamimul \\ Islam, Risalat Binte Hossain, Waseq Billah, Md Shofiul Alam
}

\begin{abstract}
Every year, tobacco industries are spending millions of dollars targeting youths and non-smokers with tactful advertisements and promotion, which has impact on early initiation, increasing overall smoking rate and further upsurge the disease and early death. The study aimed to explore the tobacco industry's branding strategies that influences youth to early initiation of tobacco in Bangladesh. This study was a cross-sectional design with mixed method approach and implemented during March - December 2019. Survey was conducted using semi-structured questionnaire through face-to-face interview among 208 students in different educational institutions of the selected areas of Dhaka city as well as twenty-five (25) KIIs were conducted with different experts personnel. Descriptive and inferential analysis were performed for quantitative data. Thematic analysis was done for qualitative data. Among student respondents, $71.0 \%$ were smokers and their average age of initiation was 13.8 years. Study revealed that $21 \%$ students attended or exposed to different types of tobacco industry's (TI) promotional programs including one-to-one/group campaign $(61 \%)$, corporate programs $(23 \%)$, seminar $(21 \%)$, career counseling $(21 \%)$ and sports event $(2 \%)$. Among them, $70.0 \%$ students received free promotional items, such as T-shirt, lighter, cap, wrist belt, free cigarette, etc. at these programs and $65 \%$ students were interested in the motivational speech given by the TI representatives. Three-fourths (75\%) were familiar with misleading branding terms such as light, tar, full flavor, filter and menthol. The initiation age of the smoking was associated with observing the arrangement of the smoking product in the retail shop $(\mathrm{p}<0.05)$, attracted to cigarette stick and packet color $(\mathrm{p}<0.05)$ and attracted to smell/flavor $(\mathrm{p}<0.05)$ and attracted to role model/ celebrity's smoking $(\mathrm{p}<0.05)$. Among retailers, 59.3\% received TI assistance including showcase decoration with brand color, receiving model box and mounting board. Besides, 59.3\% retailers mentioned promoting new brands to the non-user including students through promotional campaign, convince/persuasion, giving free samples, etc. TI is undertaking aggressive marketing and promoting brands targeting the youth around educational institutions that are ultimately contributing to early smoking initiation. A Strong monitoring system should be in place to stop direct and indirect branding and promotional activities.
\end{abstract}

Keywords: Branding- industrial tactics- early initiation- youth- digitalizing

Asian Pac J Cancer Prev, 22, Progress of Tobacco Control in the South-East Asia Region Suppl, 81-88

\section{Introduction}

Tobacco is one of the most widely used addictive substances in the world, is a plant of the genus Nicotiana and native to the Americas and historically one of the half-dozen most important crops grown by American farmers as well as widely cultivated for their leaves. The tobacco industry comprises those persons and companies engaged in the growth, preparation for sale, shipment, advertisement, and distribution of tobacco and tobacco-related products which can grow in any warm, moist environment, and it can be farmed on all continents except Antarctica.

Branding of tobacco industry products is the marketing of nicotine-containing products or use. Traditionally, the tobacco industry markets cigarette smoking, but it is increasingly branding other products, such as e-cigarettes. Products are marketed through social media, stealth marketing, mass media, and sponsorship (particularly of sporting events). Expenditures on nicotine marketing are in the tens of billions a year; in the US alone, spending was over US\$ 1million per hour in 2016. In 2003, per-capita marketing spending was $\$ 290$ per adult smoker, or $\$ 45$ per inhabitant. Nicotine marketing is increasingly regulated; some forms of nicotine advertising are banned in many countries. For that the World Health Organization recommends a complete tobacco advertising ban. The tobacco industry claims not to target children or non-smokers with their advertising and promotion. This includes with the use of branding on packaging. 
In fact, the tobacco companies also claim that packaging has no role in advertising and promotion at all and that it has no impact on overall smoking rates (Bach et al., 2018).

Every year, the tobacco industry spends billions of dollars around the globe on branding, advertising, promotion and sponsorship. The tobacco industry's manipulative marketing tactics increase consumption of its products, leading to an increase in death and disease across the world. Tobacco is responsible for 5 million deaths every year (Brand Management Promotion).

If current trends continue unchecked, it is estimated that 500 million people alive today will be killed by tobacco. Millions of the tobacco industry's customers die from tobacco related disease or quit each year, and the industry must replace them in order to survive. Industry documents reveal that the companies carefully study the habits, tastes, and desires of their potential customers and use that research to develop products and marketing campaigns. The industry is very effective in enticing new consumers, including youth and women, to start using tobacco, since they are the "replacement smokers" so important to the industry's success. The tobacco industry is now a truly global industry, investing huge sums of money in low- and middle income countries to increase the social acceptability of tobacco and tobacco companies among both adults and young people4. In most developed countries, businesses use a broad variety of marketing techniques to increase their sales, gain market share, attract new users, and retain existing customers. These techniques include product design, packaging, pricing, distribution, product placement, branding, advertising, and a variety of promotional activities.

Tobacco companies were among the earliest companies to identify and implement effective, integrated marketing strategies, and cigarettes and other tobacco products have long been among the most heavily branded and marketed consumer products in the whole world (BMA, London, 20014).

Globally, the tobacco industry spends large sums of money on advertising, sponsorship and promotion. For example, in 2014 tobacco companies spent more than US\$9 billion on advertising and promotional expenses in the United States (US) alone (Clive Bates).

As advertising restrictions have become more prominent across the globe, the industry has had to adapt and be more creative in how it advertises and branding its products, using sponsorship and different types of promotion (UNDESA, 2013). Tobacco harms the health, the treasury, and the spirit of Bangladesh. Every year, more than 161,200 of its people are killed by tobacco-caused disease. Still, more than 172,000 children $(10-14$ years old) and 24,880,000 adults (15+ years old) continue to use tobacco each day. Complacency in the face of the tobacco epidemic insulates the tobacco industry in Bangladesh and ensures that tobacco's death toll will grow every year.

In 2015 , there were adult smoking are using tobacco daily $39.8 \%$ where more are male smoker than on average in low, medium and high socio-economics status (Harvard School of Public Health; 2008). Therefore, in the same year almost $1.86 \%$ children smoking (10-14 years) are using tobacco daily where more boys smoke in Bangladesh than on average in medium-HDI countries. As well as $25.54 \%$ deaths caused by tobacco consumption in 2016 . In 2013, 31.5\% more people use smokeless tobacco in Bangladesh than on average in medium -HDI countries. The economic cost of smoking in Bangladesh amounts to 158,578 million taka. This includes direct costs related to healthcare expenditures and indirect costs related to lost productivity due to early mortality and morbidity (Marvin et al, 2006).

It is a strongly clear issue that branding, advertising and promotion influence the factors that led directly to tobacco use by adolescents, women including the initiation of cigarette smoking as well as its continuation .Tobacco companies have long argued that their marketing efforts do not increase the overall demand for tobacco products and have no impact on the initiation of tobacco use among young people; rather they argue, they are competing with other companies for market share. But as a matter of fact that tobacco companies recruit new smokers, and their advertising campaigns appeal to the aspirations of adolescents (most smokers start as adolescents or even earlier) (Perry, 2015).

For that their branding, advertising and promoting fulfills many of the aspirations of adolescents and children by effectively using themes of independence, liberation, attractiveness, adventurousness, sophistication, glamour, athleticism, social acceptability and inclusion, sexual attractiveness, thinness, popularity, rebelliousness, and being "cool".

A 2003 systematic review of the published longitudinal studies on the impact of advertising concluded "that tobacco advertising and promotion increases the likelihood that adolescents will start to smoke". Marketing efforts directed at young adults may also have an impact on tobacco initiation rates within the population of a country, in that the campaigns have been shown to encourage regular smoking and increase levels of consumption. This public position of the tobacco companies is contradicted by general marketing theory about the role of packaging as a medium for advertising and promotion (ACS, 2014).

It has been rejected by courts around the world examining the issue. It is also contradicted by the internal industry documents that have been disclosed through litigation. In contrast, the weight of the evidence from extensive and increasingly sophisticated research conducted over the past few decades shows that the industry's marketing activities have been a key factor in leading young people to take up tobacco, keeping some users from quitting, and achieving greater consumption among users (Newman, 2014).

This growing evidence has helped to spur a variety of policy interventions aimed at reducing the influence of branding and marketing on tobacco initiation as well as consumption by the tobacco companies.

To see the above situation, this study aims to describe the current state of branding of the tobacco industries as well as examine the association between influence young adults to initiate tobacco consumption in different socio-economic status at the Dhaka city in Bangladesh (Philip Moris Tobacco Company; 2017). 
Objectives of the study

The study aimed to explore the tobacco industry's branding strategies and identify issues and challenges of preventing branding influences on young adult's early initiation of tobacco in Bangladesh.

\section{Materials and Methods}

This study was a cross-sectional design with quantitative approach and implemented during March - December 2019. Survey was conducted using semistructured questionnaire through face-to-face interview among 208 students (class 9 to university 2nd year) and 91 retailers in and around (100 yards) the educational institutions of the selected areas of Dhaka city.

Twenty-five (25) KIIs were conducted with tobacco control activists, Journalist, extracurricular advisors of educational institution, representative of youth club and event management personnel. Descriptive and inferential analysis were performed for quantitative data.

Thematic analysis was done for qualitative data. Institutional and individual ethical issues was addressed during conducting this study.

\section{Results}

\section{Quantitative Survey}

Table 1 presents the unweight sample size by selected demographic characteristics of the students and retailers including area, gender and type of educational institution.

In classifying sample distribution by area, almost equal number of both students and retailer respondents were interviewed in the three study areas.

According to the type of educational institutes, about half $(51.8 \%)$ of the student respondents were from the

Table 1. Educational Institutes, Gender of the Study

\begin{tabular}{lcc}
\hline Variables & $\begin{array}{c}\text { Student } \\
\text { Number (\%) }\end{array}$ & $\begin{array}{c}\text { Retailer } \\
\text { Number (\%) }\end{array}$ \\
\hline Type of institution & & $18(19.8)$ \\
$\quad$ School & $35(16.8)$ & $18(19.8)$ \\
College & $65(31.3)$ & $58(60.4)$ \\
$\quad$ University & $108(51.9)$ & \\
Gender & & $80(87.9)$ \\
$\quad$ Male & $199(95.7)$ & \\
Female & $9(4.3)$ & $91(100.0)$ \\
Total & $208(100.0)$ &
\end{tabular}

Table 2. Current Tobacco Using Status

\begin{tabular}{lcc}
\hline Variable & Number & Percent (\%) \\
\hline Age of initiation, Mean \pm SD & $13.8 \pm 2.5$ & \\
Self-Income monthly (in BDT) & & \\
$\quad$ Having self-income & 72 & 34.6 \\
Have no Income & 59 & 28.4 \\
$\quad$ Undisclosed & 77 & 37 \\
Total & 136 & 100 \\
\hline
\end{tabular}

Table 3. Information about the Retailers

\begin{tabular}{|c|c|c|}
\hline Variable & Number & Percent $(\%)$ \\
\hline \multicolumn{3}{|c|}{ Weekly sale (BDT) from whole product } \\
\hline$\leq 5000$ & 55 & 60.4 \\
\hline$>5000$ & 36 & 39.6 \\
\hline Mean \pm SD & \multicolumn{2}{|c|}{$6505 \pm 5679$} \\
\hline \multicolumn{3}{|c|}{ Weekly sale (BDT) from tobacco product only } \\
\hline$\leq 5000$ & 64 & 70.3 \\
\hline$>5000$ & 27 & 29.7 \\
\hline Mean \pm SD & \multicolumn{2}{|c|}{$4680 \pm 3028$} \\
\hline $\begin{array}{l}\text { Number of brands available, } \\
\text { Mean } \pm \text { SD }\end{array}$ & \multicolumn{2}{|c|}{$14.6 \pm 5.4$} \\
\hline \multicolumn{3}{|l|}{ Main customer } \\
\hline Student & 54 & 59.3 \\
\hline Others* & 37 & 40.8 \\
\hline \multicolumn{3}{|c|}{ Others- Businessman, Day-labor } \\
\hline Total & 91 & 100 \\
\hline
\end{tabular}

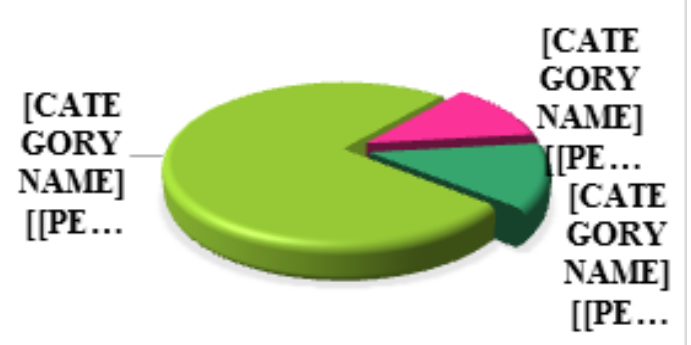

Figure 1. Residence of the Students

universities, which followed by $31.3 \%$ from College and $16.8 \%$ students were from school. Whereas, $60.8 \%$ retailer respondents were from the surrounding area of the universities (100 yards), and equal proportions (19.8\%) were from school and college, respectively. Among the respondents, $95.7 \%$ of the student respondents and $87.9 \%$ of the retailer respondents were male.

According to the individual questionnaire, all the

Table 4. Attractive Portion of the Program

\begin{tabular}{lc}
\hline Types of attractive portion* & Percent (\%) \\
\hline Motivational speech & $65.10 \%$ \\
Program arrangement & $48.80 \%$ \\
Promotional materials & $46.50 \%$ \\
Industry logo & $41.90 \%$ \\
Base, $n$ & 43 \\
\hline
\end{tabular}

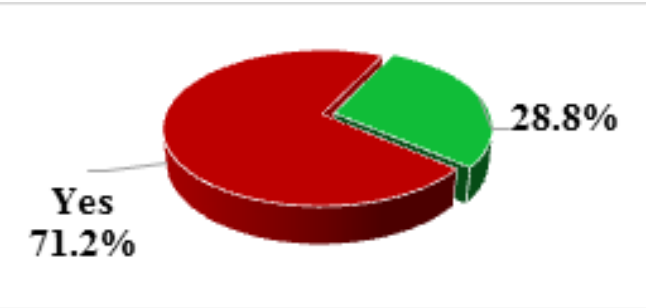

Figure 2. Current Tobacco Using Status.

Asian Pacific Journal of Cancer Prevention, Vol 22 


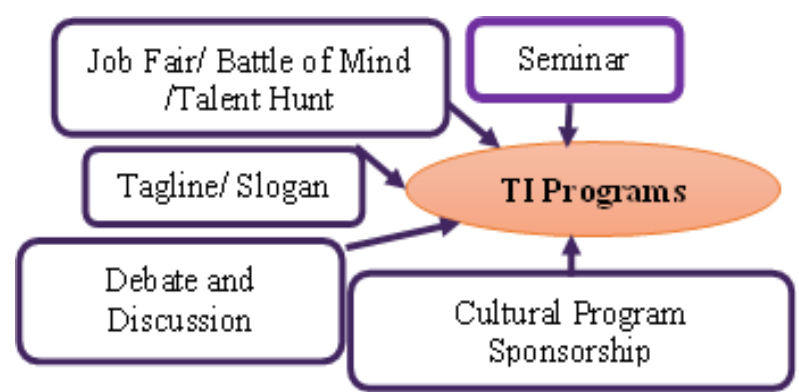

Figure 3. Tobacco Industries Bbrand Promotion through Different Types of Program

Table 5. Getting any Benefit from the Tobacco Company for Selling a New Product

\begin{tabular}{lcc}
\hline Variable & Number & Percent \\
\hline Getting benefit for selling new product & & \\
Yes & 44 & $48 \%$ \\
No & 47 & $52 \%$ \\
Types of benefit received & & \\
Tobacco product & 19 & $43 \%$ \\
Cash & 25 & $57 \%$ \\
Total & 91 & 100 \\
\hline
\end{tabular}

student respondents asked on their main residence. For that table 3.4 shows that the high proportion of the student (75\%) lived with their parents or relatives in this study. Moreover, there were one third portion (25\%) students lived in the mess and hostel or hall respectively in this report.

The prevalence of smoking tobacco is presented by "current status of tobacco use" and "non-user" among the several categories of students. Overall, three fourth (71.2\%) students used tobacco by various demographic and socioeconomic characteristics as well as $28.8 \%$ did not use any kind of tobacco in this study.

According to the Table no 2 shows that the average

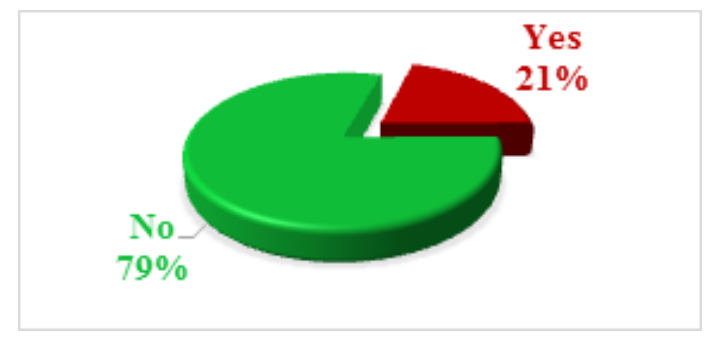

Figure 4. Attending Any Program Arranged by TI
Table 6. The Following Display which have in the Retail Shop

\begin{tabular}{lc}
\hline Display in the retail shop & Percent (\%) \\
\hline Poster & $85.6 \%$ \\
Leaflet & $78.9 \%$ \\
Product Display & $76.7 \%$ \\
Model Box & $61.1 \%$ \\
Cartoon Display & $51.1 \%$ \\
Showcase with Color & $45.6 \%$ \\
Decorated with Logo and color & $48.9 \%$ \\
Banner and Signboard & $36.7 \%$ \\
Showcase with Logo & $35.6 \%$ \\
Umbrella & $23.3 \%$ \\
Glass box & $13.3 \%$ \\
Base, $\mathrm{n}$ & 90 \\
\hline
\end{tabular}

age of initiation of smoking product among the youth was thirteen years old to find in this study. On the contrary, several categories reported in this study were merged to consider five exclusive self-monthly income which are less than three thousand, three thousand to ten thousand nine hundred ninety, more than eleven thousand and Have no Income, Not Interested etc. Usually, the highest proportion of $28.4 \%$ students had no self-monthly income, $37 \%$ students did not interest to share about it in this report. Additionally, the proportion of $34.7 \%$ students' self-monthly income were less than three thousand to more than eleven thousand taka. Therefore, one an average their self-monthly income was six thousand four hundred one to find in this report.

According to the table no 3 shows that quite a few sorts stated in this study were merged to consider two exclusive weekly sell from the whole products and tobacco product only. Overall, 60.4\% retailer respondents sold weekly earned less than five thousand from the whole products in their shop. Consequently, one an average their

\begin{tabular}{ll}
\hline & \\
& \\
& \\
& \\
& \\
& \\
Figure 5. Receiving Any Free Promotion Items from the \\
Program \\
n-user)
\end{tabular}

Table 7. The Most Attracting Part of the Product (user and non-user)

\begin{tabular}{lll}
\hline Branding terms* & Students' Perception & Percent (\%) \\
\hline Filter & Nicotine control, safer/ less harmful & $66.20 \%$ \\
Ultra-light / Ultra-low Tar & Safer/ less harmful, less nicotine/tar, soft & $13.50 \%$ \\
Full Flavour/ Regular & Deep flavor, More Nicotine & $10.80 \%$ \\
Lights/Mild/Low Tar & Light flavor, Safer/ less harmful, less nicotine/tar, soft & $9.50 \%$ \\
Menthol & Cooler/ smooth feeler, Sweetness & $6.80 \%$ \\
Base, $\mathrm{n}$ & & 157 \\
\hline
\end{tabular}


Table 8 .

\begin{tabular}{lc}
\hline & Percent \\
\hline Attractive part & \\
Brand Name and Logo & $36.00 \%$ \\
Color of cigarette sticks and packaging & $30.70 \%$ \\
Packet Design/size & $29.70 \%$ \\
Base, $\mathrm{n}$ & 208 \\
Attractive part (Users) & \\
Smell & $19.00 \%$ \\
Flavor & $39.70 \%$ \\
Base, $\mathrm{n}$ & 147 \\
\hline
\end{tabular}

weekly profit from the whole profit was six thousand five hundred six to find in this report. On the contrary, the highest proportion of $70.3 \%$ retailer's weekly profit from the tobacco products only were less than five thousand Additionally, almost $30 \%$ retailers sold more than five thousand taka from the tobacco products weekly. As a result, one and average their sale was four thousand six hundred eighty to find in this report. Table 3 also shows that there was come different types of customer in the retail shop to buy these product by retailers. Overall, 59.3\% retailers sold smoking tobacco product in different brands to the students as the sampling area was selected a few different types of educational institutes crowded nearby the available retail shop which is the highest percentage in this report; $41 \%$ retailer respondents sold smokeless tobacco product in different brands to the others customer which were arranged in the eye level of the customers (several profession) customers in this study. On an average, they sold almost fifteen different types of several brand of smoking product to the customer every day.

\section{Brand Promotion [Program by Tobacco Industry (TI)]}

There were arranged various types of program of the tobacco industries for brand promotion of their smoking tobacco product in the different area in Bangladesh. They arranged job fair and seminar in the several public and private educational institutes like university as well as tactfully promoted their company by their company logo and different types of promotional incentives and made a good image among the students according to the interviewer's information. In addition, the tobacco company organized annual concert, cultural program for their corporate employees, wholesalers, retailers, staffs of different precious venue in the Bangladesh to motivate and refreshment of their all types of employee to work faithfully with them to earn more profit of their company and promote their smoking product in different brands in here. In these programs they used several motivational and catchy tagline or slogan to attract the customers or audiences. Furthermore, the tobacco industries arranged debate discussion, award program, talk-show and documentary program which was seen mass media, print media and social media about their successful career or good things of the tobacco company to motivate the general audience and increased their face value among the audience especially to the youth according to the majority of the interviewers information's. According to this study the table number 4 showed that $79 \%$ student did not attend any program which was arranged by the tobacco company but $21 \%$ attended in the several program arrange. In the above Figure 4 showed that $14.4 \%$ students received different types of free promotional item such as T-shirt, wrist belt, caps. Bags etc. from this program as well as $30 \%$ did not received it. According to the Table number 4 showed that there were many thing to attract this programs among the students which were merged to consider four exclusive categories including industry logo, program arrangement, motivational speech, promotional materials etc. Normally, $41.9 \%$ students attracted to see their industry logo, $48.8 \%$ respondents attracted to their program arrangement, $65.1 \%$ attracted or motivated to listen their motivational speech which was the highest proportion of the study as well as $46.5 \%$ students concerned to get their promotional materials from them which was also related to make a good image about the tobacco industries among the students respondents. As stated by the figure 5 showed that $44.0 \%$ retailer respondents got target from the tobacco company for selling smoking tobacco product but $56 \%$ retailer respondents did not get target from the tobacco company for selling smoking tobacco product. According to the table number 5 showed that $48 \%$ retailer respondents got benefits from the tobacco company for selling a new product but $52 \%$ retailer respondents did not get benefits from the tobacco company for selling a new product. For that the retailers who received different types of benefit from the company for selling a new product including cash, tobacco product etc. Generally, 57\% retailer respondents got cash from the tobacco company and $43.2 \%$ retailer respondents received tobacco product which was also related to make a faithful business image about the tobacco industries among the respondents. In this table represented the displaying product in the retail shop which were provide by the tobacco company directly. Generally, 35.6\% retailers had showcase with logo to get from the tobacco company to set/decorate shop, $45.6 \%$ had showcase with color, $61.1 \%$ retailer had model box, $23.3 \%$ got umbrella, $13.3 \%$ received glass box, $78.9 \%$ received leaflet, $85.6 \%$ retailers got poster to show in their retail shop, $15.6 \%$ got signboard, $21.1 \%$ received banner, $51.1 \%$ got cartoon display to set/decorate shop, $22.2 \%$ retailers decorated with color in their shop, $26.7 \%$ retailer respondents decorated with logo in their shop and $76.4 \%$ retailer respondents received product display from the tobacco company to set/decorate shop and thus the tobacco company continue their evil business nearby different types the educational institutes to explore in this study.

\section{Product and Personal Branding [Among Students]}

According to the table no 8 showed that $36 \%$ student attracted to the brand name and logo of the tobacco company, $31 \%$ student attracted to see the packet color in the cigarettes, $30 \%$ attracted to see the packet design or size, $19 \%$ attracted to see the cigarette smell, $40 \%$ attracted on the cigarette flavor which was a great findings in the study.

According to this study the table 8 also showed that there were different familiar branding terms and knowledge about the meaning of the familiar branding 
terms among the students. Overall, 66.2\% students knew 'Filter' as a familiar branding terms which mean that Extra portion, Flavor, Less nicotine, More Nicotine, Safer/ less harmful'. 13.5\% students knew 'Ultra-light / Ultra-low Tar' as a familiar branding terms which mean that Safer/ less harmful, less nicotine, less tar, soft, Define feel, Light flavor' as well 10.8\% students knew 'Full Flavor/ Regular' as a familiar branding terms which mean that Extra portion, Flavor, Less nicotine, More Nicotine, Safer/ less harmful'. Similarly, 9.5\% students knew 'Lights/Mild/Low Tar' which mean that Less nicotine, less tar, soft, Average Deep flavor, More Nicotine' as a familiar branding terms and and only $6.8 \%$ students knew 'Menthol' as a familiar branding terms which means that Hunger, Purity, Sweetness, cooler/ smoother feeler' was the lowest proportion of the study to find in this study.

\section{Discussion}

The results of this present study suggested that majority of the student were smokers (71\%) and $97.3 \%$ respondents consumed different types of smoking products where cigarettes were more consuming tobacco products among them and $84 \%$ took cigarettes regularly in this study as the POS were available of their educational institutes which is an important reason behind to initiate smoking. According to the study showed that $66.2 \%$ smoker students switched different types of brand due to unavailability, increased price, new flavor, cigarette stick color and uphold status etc. But $33.8 \%$ students did not switch brand due to availability, fix price, flavor, and uphold status etc.

In fact, the average age of initiation of smoking product among the youth was thirteen years old to find in this study which was also shifted from the previous study which is found in this study due to availability, affordability, and different types of promotional activities of the tobacco company. Agreeing to the study, there were an influencing issue between the initiation age of the smoking (users) by different types of educational institute where $48.6 \%$ school students started smoking when they were up to twelve years old which followed by $47.1 \%$ college students started smoking when they were thirteen to fourteen years old. Additionally, 46.2\% university students started smoking when they were more than fifteen years old.

The result submitted that $21 \%$ student attended at any program which was arranged by the tobacco company where $60.5 \%$ students presented in the campaign at any place like $62.9 \%$ students joined the program which held at their own institutes and $4.6 \%$ students joined in others place which arranged by the tobacco company and 70\% students received different types of free promotional item from this program and $30 \%$ did not receive them from this program in this study. Along with the study, there were a relation between the initiation age of the smoking (users) by receiving any free promotion item from this program among the students where $40 \%$ students received any free promotion item from the mentioning program on the above table at the age of eleven to sixteen years old to influence the initiation of smoking.

There were arranged various types of program of the tobacco industries for brand promotion of their smoking tobacco product in the different area in Bangladesh. Most of interviewer gave their opinions about it and also told that the supply transports of the smoking tobacco products were used to more attractive or eye catching color or color gradient like dark pink, sky blue, orange, magenta, purple etc. which are prompt and familiar easily among the customer especially youth. Moreover, there were used the unit and special motivational quotation in these transports which were responsible to influence to the initiation of the smoking tobacco among the customer especially students. Even though, these vehicle were often stood in front of the retail shop or point of sell in the Dhaka city.

They also arranged job fair and seminar in the several public and private educational institutes like university as well as tactfully promoted their company by their company logo and different types of promotional incentives and made a good image among the students according to the interviewer's information in the qualitative findings.

Moreover, the findings also showed that $30 \%$ students got $\mathrm{t}$-shirt as a promotional item from the tobacco company, $20 \%$ got cap, $6.7 \%$ got wrist belt from them and similar to get bag and received ticket from them for different types of offering issues and $30 \%$ got others issues from this promotional program which was related to the initiation of the smoking among the respondents.

At the same time, $65.1 \%$ attracted or motivated to listen their motivational speech which followed by $48.8 \%$ respondents attracted to their program arrangement, $41.9 \%$ students attracted to see their industry logo, and $46.5 \%$ students concerned to get their promotional materials.

According to the study showed that $48.4 \%$ retailer respondents got benefits from the tobacco company for selling a new product but $51.6 \%$ retailer respondents did not get benefits from the tobacco company for selling a new product. For that, $56 \%$ retailer respondents got cash from the tobacco company and $43.2 \%$ retailer respondents received tobacco product which was also related to make a faithful business image and inspire to influence to the initiation of the smoking tobacco about the tobacco industries among the respondents as well as $44.0 \%$ retailer respondents got target from the tobacco company for selling smoking tobacco product where $43.9 \%$ retailers received cash from the tobacco company to fulfill the target, $46.3 \%$ got tobacco products, from the tobacco company to fulfill target and thus the tobacco company continue their successful business nearby different types the educational institutes to explore in this study.

According to the interviewer's opinion, the tobacco company gave incentives to the customer when a new product launched or others issues as well as also gave to the retailers for several time on their performance to make a good profit. Moreover, Tobacco Company gave target sell at a specific age group through retailer to follow their industries policy which is responsible to early initiation of the smoking tobacco. They also provided poster, leaflet and placard of the different brand of smoking tobacco products with different color company name, logo and design which put in front of the customer to attract them.

In addition, the tobacco company arranged campaign and cultural events for their new/ old product and business 
purpose through brand representation to use an especial dress-code with tobacco company name and logo which is influenced to initiated smoking at an early age.

As stated by the report showed that $59.3 \%$ retailer respondents received any support from the company to set/decorate shop for selling smoking tobacco product and many things to get incentives for support from the tobacco company to set/decorate shop among the retailers like: Showcase, Model Box, Signboard, Banner, Cash, Decoration materials, Technical support for decoration and others etc.

According to the study showed that there were many attractive things to observe through display of the Smoking tobacco products in the retail shop where $60.0 \%$ students attracted to the cigarette stick color and packet color of the smoking tobacco product in the retail shop when their smoking initiation age was up to twelve years old which followed by $28.6 \%$ students attracted to the different types of cigarette flavor and smell of the smoking tobacco product more than fifteen years old in the retail shop.

In the meantime, $36 \%$ student attracted to the brand name and logo of the tobacco company, $31 \%$ student attracted to see the packet color in the cigarettes, 30\% attracted to see the packet design or size, $19 \%$ attracted to see the cigarette smell, $40 \%$ attracted on the cigarette flavor which was a great findings in the study.

On the word of this study also showed that there were different familiar branding terms among the student which were merged to consider seven exclusive categories where overall, $66.2 \%$ students knew 'Filter' as a familiar branding terms which mean that Extra portion, Flavor, Less nicotine, More Nicotine, Safer/ less harmful'. 13.5\% students knew 'Ultra-light / Ultra-low Tar' as a familiar branding terms which mean that Safer/ less harmful, less nicotine, less tar, soft, Define feel, Light flavor' as well 10.8\% students knew 'Full Flavor/ Regular' as a familiar branding terms which mean that Extra portion, Flavor, Less nicotine, More Nicotine, Safer/ less harmful'. Similarly, 9.5\% students knew 'Lights/Mild/Low Tar' which mean that Less nicotine, less tar, soft, Average Deep flavor, More Nicotine' as a familiar branding terms and and only $6.8 \%$ students knew 'Menthol' as a familiar branding terms which means that Hunger, Purity, Sweetness, cooler/ smoother feeler' was the lowest proportion to find in this study which are directly influence to initiate tobacco among the youth.

As the state of interviewers, the tobacco company prepared lots of poster, leaflet, placard, banner, brochures with attractive color spectrum, brand name and company logo which are brought to the point of sell and retail shops to set in the shop at the eye level of the customer to attract them and related to the initiation of the customer specially youth.

Furthermore, Tobacco Company fixed the level of chemical adjustment of the cigarettes like nicotine, tar, benzene, ammonia etc. so that the customer attract and addicted to the smoking product as well as also determined the arrangement of the Pattern of the Smoking Brand on the display (Eye Level) in the retail shop and fixed target group of customer (14-15years) among the retailers surrounding the educational institutes as the state of the interviewers. For that reasons, they provided various motivational incentives among the retailers to make a good profit according to the interviewers' opinion to find the qualitative findings.

\section{Conclusion and Recommendation}

The study indicated that almost all of the different age groups respondents had knowledge about the tobacco product in different brands from point of sell and internet among all types of students (like smokers and non-smokers). As well, majority of the student were smokers and consumed different types of smoking products like cigarettes regularly in this study as the POS were available of their educational institutes which is an important reason behind to initiate smoking.

On the contrary, half of the students started their smoking when they age were less than eleven years old due to availability, affordability, and different types of promotional activities of the tobacco company.

There were another findings that the most commonlymentioned issue was concerned about the purchasing pattern of the cigarettes among the students where majority of the students took single stick casually from the nearby POS, retail shops and others availability which is out of control to parents, educational authority, society and also community due to lack of awareness, weekly imposed law and less monitoring of the family and concerns etc.

Consequently to these reasons, the tobacco company used to lack and gaps of us and make their evil successful business to influence these youth to initiate smoking at an early age by doing different types of promotional activities like seminars, career counselling, campaigns, cultural events etc. where majority of the student be presented at these programs and the tobacco company provided them different types of promotional items to make a good image among the students about their mechanism towards involve a new customer for a long time.

In the meantime, tobacco industries prepared lots of poster, leaflet, placard, banner, brochures with attractive color spectrum, brand name and company logo which are brought to the point of sell and retail shops to set in the shop at the eye level of the customer to attract them and related to the initiation of the customer specially youth.

Furthermore, Tobacco Company fixed the level of chemical adjustment of the cigarettes like nicotine, tar, benzene, ammonia etc. so that the customer attract and addicted to the smoking product as well as also determined the arrangement of the Pattern of the Smoking Brand on the display (Eye Level) in the retail shop and fixed target group of customer (14-15years) among the retailers surrounding the educational institutes as the state of the interviewers. For that reasons, they provided various motivational incentives among the retailers to make a good profit according to the interviewers' opinion to find the qualitative findings.

However, there were most of the retail shop and point of sell and hawkers were available surroundings to the educational institutes where the students accessed easily to buy smoking tobacco product at any age and these smoking tobacco product sold a reasonable price of different flavor in various brands which were displayed 
at their eye level on the model box in the retail shop and it was directly responsible to the early initiation of the smoking tobacco among the students.

There is considerable discussion in the public health community about the role of potentially less harmful tobacco products. This issue is complex. Moreover, there is no substitute for the weight of government action when it comes to implementing these proven measures.

As young generation is more susceptible to adopt different brands of tobacco and it is likely to changes their behavior which tumbled them into early initiation of smoking which should be more concerned to the family, society, community and The government of the Bangladesh etc.

Therefore, strong policy must be initiated to prevent these young Bangladeshi generation from such addictive behavior and early initiation of tobacco.

Tobacco industry's promotional activities, indirect advertisement, tactful advancement through social media, using other platform can be negatively influenced for increasing usage of tobacco by the youth day-by-day.

The above scenario should be controlled strictly by rules and regulations of the government in Bangladesh. So, this study results would help to document information and suggests to develop proper interventional measures to stop this kind of tactful branding of tobacco companies.

\section{Author Contribution Statement}

This review was carried out and approved in collaboration between all the authors. USA conceptualized and developed the study protocol. USA, HMMM, AIN, $\mathrm{RBH}, \mathrm{WB}, \mathrm{MSA}$ and MSI identified records for full text review and data extraction. USA and HMMM drafted the manuscript.

\section{Acknowledgements}

The publishing cost was provided by WHO office in South-East Asia

\section{References}

Bach L (10 April 2018). "Tobacco Product Marketing on the Internet” (PDF), Campaign for Tobacco-Free Kids, retrieved 2018-05-25.

Brand Management Promotion; Tutorial points, simply easy learning.

British Medical Association. Forever Cool: The influence of smoking imagery on young people. BMA, London, 20014.

Clive Bates and Andy Rowell for the London-based Action on Smoking and health (ASH).; Tobacco Explained "The truth about tobacco industry.... In its own words".

Definition of Youth; Website of the Focal Point on Youth, UNDESA, 2013, 1-23; Microsoft Word Youth Definition_2013-1 23.

Harvard School of Public Health; July 18, 2008; Tobacco Industry Manipulated Cigarette Menthol Content To Recruit New Smokers Among Young People, Research Shows.

Jump up to:a b 1999 advertisement, in the collection of Stanford Research Into the Impact of Tobacco Advertising.

Marvin E Goldberg, Ronald M Davis, and Anne Marie O'Keefe;
Tob Control. 2006 Dec; 15, The role of tobacco advertising and promotion: themes employed in litigation by tobacco industry witnesses.

"Manufacturing Cigarettes" World Lung Foundation and American Cancer Society. Archived from the originalon May 24, 2012. Retrieved June 7, 2014.

Newman AA (2014-08-10). “A Less Defiant Tack in a Campaign to Curb Smoking by Teenagers". The New York Times. Retrieved 2018-05-24.

Perry CL How do you sell death; 2015.

Personal Branding: How to Successfully Build Your Brand by Michael Stelzner, September 2019 Book.

Rapa port L (6 April 2018) "More U.S. teens seeing e-cigarette ads". Business Insider. Retrieved 2018-05-18.

The Tobacco atlas 6th editions with Bangladesh context, 2018.

Tobacco Pack Branding-Philip Moris Tobacco Company; Theory and Pratice; 2017.

“Tobacco Leaf Harvesting, Curing, and Fermenting” Leaf Only. Retrieved 20 April 2017.

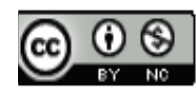

This work is licensed under a Creative Commons AttributionNon Commercial 4.0 International License. 